smokers $(\beta=-3.7, p=0.006)$ while on average the DLCO/VA decreased from 78.1 (SD 18) to 76.5 (SD 17). Changes in FVC were however not associated with smoking behaviour $(\mathrm{p}=0.13)$.

The mRSS changed between baseline and follow-up from an average of 7.6 (SD 7) to 7.3 (SD 7); on average, in smokers the mRSS improved faster than in never smokers $(\beta=-0.65, p=0.015)$. The presence of DUs at follow-up was not associated with current smoking $(\mathrm{p}=0.9)$.

Having previously smoked was not significantly associated with the progression of any of the assessed outcomes.

Conclusions: Although the adverse effects of smoking on bronchial airways that is known in the general population could be replicated in the SSc population, the lack of a measurable adverse effect of smoking on SSc organ manifestations argues against a major role of tobacco associated free radicals and vasoconstriction in the pathogenesis of SSC vasculopathy and fibrosis.

Disclosure of Interest: V. Jaeger: None declared, G. Valentini: None declared, E. Hachulla: None declared, L. Czirják: None declared, E. Siegert: None declared, O. Distler Grant/research support from: 4 D Science, AbbVie, Actelion, Active Biotec, Bayer, Biogenldec, BMS, Boehringer Ingelheim, ChemomAb, EpiPharm, espeRare foundation, Genentech/Roche, GSK, Inventiva, iQone Healthcare, Lilly, medac, Mepha, Medlmmune, Mitsubishi Tanabe Pharma, Pharmacyclics, Pfizer, Sanofi, Serodapharm and Sinoxa, Consultant for: 4 D Science, AbbVie, Actelion, Active Biotec, Bayer, Biogenldec, BMS, Boehringer Ingelheim, ChemomAb, EpiPharm, espeRare foundation, Genentech/Roche, GSK, Inventiva, iQone Healthcare, Lilly, medac, Mepha, Medlmmune, Mitsubishi Tanabe Pharma, Pharmacyclics, Pfizer, Sanofi, Serodapharm and Sinoxa, L. M. Bambara: None declared, E. Rosato: None declared, Y. Allanore: None declared, M. MatucciCerinic: None declared, P. Airó: None declared, U. Walker: None declared DOI: 10.1136/annrheumdis-2017-eular.2729

\section{SAT0367 CAPILLAROSCOPY PATTERNS AMONG SYSTEMIC SCLEROSIS PATIENTS WITH GASTROINTESTINAL INVOLVEMENT AND MALNUTRITION EVALUATED BY SELF-REPORTED QUESTIONNAIRES}

Y. Yalçınkaya, Z. Ertürk, A.U. ÜNal, S. Kaymaz, P. Atagündüz, H. Direskeneli, N. Inanç. Department of Internal Medicine, Division of Rheumatology, Marmara University, School of Medicine, Istanbul, Turkey

Background: Gastrointestinal system (Gl) is commonly involved in systemic sclerosis (SSc) beginning after early stages of disease.

Objectives: We aimed to investigate capillaroscopic findings among the SSc patients with $\mathrm{Gl}$ involvement and malnutrition.

Methods: Gl involvement was evaluated by UCLA SCTC GIT 2.0 questionnaire (Khanna D.) (7 multi-item; reflux, distension, soilage, diarrhea, social functioning, emotional wellbeing and constipation) in SSc patients ACR/EULAR classification criteria (2013). To report the nutrition status of the patients "malnutrition universal screening tool (MUST)" (sum of the scores of body mass index, weight loss in last 3-6 months and acute disease effect) scores were calculated. Simultaneously, nail fold video-capillaroscopy (NVC) was performed in all paatients to deterimine ealy, active late scleroderma patterns (Cutalo et al.).

Results: In 58 SSc patients (51 female); the mean age, duration of Raynaud's, non-Raynaud symptom (year) and follow-up (month) were $46 \pm 13,54 \pm 50,10 \pm 9$ and $7 \pm 7$, respectively. Limited cutaneous form, positive ANA and anti-Scl70, telangiectases, dysphagia, digital ulcers, lung disease were found in $40(71 \%)$, $48(83 \%)$ and $21(36 \%), 45(78 \%), 39(67 \%), 33(57 \%), 23(40 \%)$ in patients, respectively. Of the NVC patterns, early was found in $16(28 \%)$, active was in $11(19 \%)$, late was in $28(48 \%)$ and normal in $3(5 \%)$. The scores of disease activity, severity and Gl were shown to be higher in patients with late NVC pattern (table-1). Of the patients with MUST score of $>0(n=10), 9$ had late and 1 had early NVC pattern.

Table 1. The scores of disease activity and severity and UCLA SCTC GIT 2.0 in SSc patients

\begin{tabular}{lcccc}
\hline & NVC (early) & NVC (active) & NVC (late) & $\mathrm{p}$ \\
\hline Modified Rodnan skin score & $4,3 \pm 5,1$ & $8,3 \pm 6,9$ & $12,3 \pm 7,5$ & $\mathrm{P}=0,001^{*}$ \\
Telangiectasia score (Shah) & $2,3 \pm 2,4$ & $4,6 \pm 1,9$ & $6 \pm 3,5$ & $\mathrm{P}<0,001^{*}$ \\
Disease activty score (Valentini) & $0,9 \pm 0,9$ & $1,1 \pm 1,2$ & $1,9 \pm 1,2$ & $\mathrm{P}=0,014^{*}$ \\
Disease severity score (Medsger) & $3,4 \pm 2$ & $4,6 \pm 3$ & $6,5 \pm 2,6$ & $\mathrm{P}=0,001^{*}$ \\
UCLA SCTC GIT 2.0 & $0,063 \pm 0,111$ & $0,259 \pm 0,288$ & $0,353 \pm 0,327$ & $\mathrm{P}=0,002^{*}$ \\
Reflux & $0,263 \pm 0,415$ & $0,500 \pm 0,509$ & $0,874 \pm 0,667$ & $\mathrm{P}=0,002^{*}$ \\
Distension & $0,118 \pm 0,357$ & $0,296 \pm 0,498$ & $0,564 \pm 0,881$ & $\mathrm{NS}$ \\
Soilage & $0 \pm 0$ & $0 \pm \pm 0$ & $0,107 \pm 0,315$ & $\mathrm{NS}$ \\
Diarrhea & $0 \pm 0$ & $0,500 \pm 0,741$ & $0,268 \pm 0,552$ & $\mathrm{NS}$ \\
Social functioning & $0 \pm 0$ & $0,105 \pm 0,237$ & $0,184 \pm 0,406$ & $\mathrm{NS}$ \\
Emotional wellbeing & $0,011 \pm 0,050$ & $0,211 \pm 0,372$ & $0,118 \pm 0,308$ & $\mathrm{NS}$ \\
Constipation & $0,184 \pm 0,380$ & $0,159 \pm 0,322$ & $0,259 \pm 0,469$ & $\mathrm{NS}$ \\
\hline
\end{tabular}

${ }^{*}$ Bonferroni, NS = not significant.

Conclusions: This SSc cohort predominantly had limited cutanous disease and vascular manifestations. In patients with late scleroderma pattern, severity of Gl was higher similar to disease activity and severity. Undernutrition was frequently seen in patients with late pattern. NVC might be useful to predict the severity of $\mathrm{GI}$ and malnutrition and allow to direct the patients to required procedures earlier. Disclosure of Interest: None declared

DOI: 10.1136/annrheumdis-2017-eular.5394

\section{SAT0368 THE PREVALENCE OF OSTEOMYELITIS IN INFECTED DIGITAL} ULCERS IN SYSTEMIC SCLEROSIS PATIENTS

Y. Braun-Moscovici ${ }^{1}$, Z. Keidar ${ }^{2}$, M. Braun ${ }^{3}$, D. Markovits ${ }^{1}$, K. Toledano ${ }^{1}$,

Y. Tavor ${ }^{1}$, F. Sabbah ${ }^{1}$, A. Rozin ${ }^{1}$, K. Dolnikov ${ }^{1}$, A. Balbir-Gurman ${ }^{1}$.

${ }^{1}$ Rheumatology Department, Rambam Health Care Campus, Rappaport Faculty of Medicine, Technion; ${ }^{2}$ Department of Nuclear Medicine, Rambam Health Care Campus Rappaport Faculty of Medicine Technion, Haifa; ${ }^{3}$ Liver Institute,

Beilinson Hospital Petach-Tikva, Sackler School of Medicine, Tel Aviv University, Petach-Tikva, Israel

Background: Skin ulcers, particularly digital ulcers occur in at least $50 \%$ of systemic sclerosis (SSc) patients (pts) and cause significant morbidity. They are often complicated by local infection which can lead to contiguous osteomyelitis.

Objectives: Our aims were to evaluate the accuracy of clinical diagnosis of osteomyelitis and to assess whether there are clinical parameters that may improve the precision of the clinical diagnosis.

Methods: We retrospectively analyzed the clinical data of consecutive SSc patients hospitalized for skin ulcers in a tertiary referral center for SSc. Our cohort is part of the EUSTAR cohort. The patients were evaluated by rheumathologists skilled in managing SSc skin lesions. All the patients with infected ulcers and suspected for contiguous involvement of underlying bone had bone scans. All the positive scans were followed with $99 \mathrm{~m}$-Technetium-Tc-labeled white blood cells (WBC) scintigraphy, in order to differentiate true osteomyelitis from acro-osteolysis or soft tissue infection. We collected demographic data, disease type, extent and severity, routine lab data (CBC, C-reactive protein -CRP, erythrocyte sedimentation rate (ESR), alkaline phosphatase (ALKP), albumin) and wound culture. Each hospitalization was considered a separate event. Statistical analysis: descriptive, student's T test, Mann-Whitney test.

Results: During the years 2003-2016, 220 SSc pts with skin/digital ulcers were hospitalized in our department for ilomedin treatment (993 hospitalizations). Most of the pts were hospitalized several times due to recurrent ulcers. Tc bone scan was performed in 39 pts (59 admissions) with infected ulcers (32 females, mean (SD) age 48 (15), disease duration 9 (6.6) years, 25 with diffuse SSc, skin score (MRSS) 9.9 (8)). Osteomyelitis was confirmed in 18 pts on 23 occasions. Osteomyelitis occurred twice in 5 pts in different locations. No statistically significant differences were found between the group with positive scans and the group with negative scans regarding demographic, clinical and lab data. 9 pts had 25 admissions for infected ulcers, osteomyelitis was confirmed in 14 of the admissions. No statistically significant differences were found for CBC ESR CRP ALKP between the osteomyelitis events and superficial infected ulcer admissions in these pts. The causative infectious agents were similar between the 2 groups. Positive bone and WBC scans confirmed osteomyelitis in $39 \%$ of clinical suspected cases. WBC scans confirmed osteomyelitis in $75 \%$ or the patients with positive Tc bone scans.

Conclusions: The prevalence of osteomyelitis among our SSc pts admitted for digital ulcers was $10 \%$. The prevalence of confirmed osteomyelitis by scintigraphy in clinically highly suspected cases was $39 \%$. Even when contiguous osteomyelitis was suspected by highly skilled rheumathologists, bone scan ruled out the diagnosis in $61 \%$ of the cases, thus avoiding unnecessary prolonged antibiotic therapy. No clinical predictors to rule osteomyelitis in or out could be identified.

Disclosure of Interest: None declared

DOI: 10.1136/annrheumdis-2017-eular.6474

\section{SAT0369 HETEROGENEITY IN THE UNDERLINING PATHOGENESIS IN PATIENTS WITH CONNECTIVE TISSUE DISEASE-ASSOCIATED BORDERLINE MEAN PULMONARY ARTERIAL PRESSURE AND ITS DISTINCTIVE HEMODYNAMIC CHARACTERISTICS FROM THOSE WITH NORMAL PULMONARY ARTERIAL PRESSURE}

Y. Yamasaki ${ }^{1}$, Y. Asari ${ }^{1}$, K. Tsuchida ${ }^{1}$, K. Suzuki ${ }^{2}$, Y.J. Akashi ${ }^{2}$, T. Okazaki $^{2}$, S. Ozaki ${ }^{1}$, H. Yamada ${ }^{3} .{ }^{1}$ Rheumatology and Allergology; ${ }^{2}$ Cardiology, St. Marianna University School of Medicine, Kawasaki; ${ }^{3}$ Medical Center for Rheumatic diseases, Seirei Yokohama Hospital, Yokohama, Japan

Background: Borderline mean pulmonary artery pressure (PAP) $(21-24 \mathrm{mmHg})$ may comprise a transition phase from normal pulmonary hemodynamic condition to pulmonary arterial hypertension (PAH), which is one of the fatal complications in connective tissue diseases (CTD) [1]. The accumulated evidence is not enough to conclude that treatment for PAH in this stage may be associated with improving clinical outcomes. On the other hand, CTD-associated PH is caused by various cardiopulmonary comorbidities. It is possible that borderline mean PAP, like overt $\mathrm{PH}$, is caused not only by pulmonary vasculopathy but also by cardiac and pulmonary complications [2].

Objectives: To clarify whether patients with borderline mean PAP associated with CTD have distinctive hemodynamic characteristics from those with normal mean PAP and its underlining pathogenesis is heterogeneous as those with manifest $\mathrm{PH}$.

Methods: We retrospectively investigated 76 CTD patients who underwent right heart catheterization from 2008 through 2016. Of the 76 patients, $25(33 \%)$ and $16(21 \%)$ had manifest PH and borderline mean PAP, respectively. The rest of the 35 patients had normal mean PAP. Systemic sclerosis was the most common 
CTD among the enrolled patients (61\%), followed by MCTD (16\%). Hemodynamic characteristics and findings after exercise echocardiography in patients having normal mean PAP, borderline mean PAP, and PH were compared. We also determined the validity of therapeutic intervention.

Results: The values of tricuspid regurgitation pressure gradient (TRPG) were comparable between patients with borderline $(31.1 \pm 7.8 \mathrm{mmHg})$ and normal mean PAP $(28.3 \pm 6.9 \mathrm{mmHg})(\mathrm{P}=0.1572)$ but its value became significantly higher in patients with borderline mean PAP $(39.1 \pm 8.0 \mathrm{mmHg})$ than in those with normal mean PAP $(32.8 \pm 7.4 \mathrm{mmHg})$ after exercise echocardiography $(\mathrm{P}=0.0391)$. Pulmonary arterial wedge pressure was significantly elevated in patients with borderline mean PAP $(12 \pm 3 \mathrm{mmHg})$ compared with that in normal mean PAP $(7 \pm 3 \mathrm{mmHg})(P<0.0001)$ and its value was comparable to those with overt $\mathrm{PH}$, suggesting the heterogeneity on the cause of borderline mean PAP among CTD patients. The clinical course of 10 patients with borderline mean PAP was studied. Five were treated for precapillary disease, 3 for postcapillary disease, and 2 for interstitial lung disease (ILD). Normalization of mean PAP was seen in $3 / 4$ and $3 / 3$ of the patients treated for precapillary and postcapillary disease, respectively. Deterioration of TRPG was seen in one patient after receiving pulmonary vasodilators. One with severe ILD developed PH.

Conclusions: The pathogenesis of borderline mean PAP, clearly distinctive from normal mean PAP, was heterogeneous as that of manifest PH in CTD patients. Though the clinical course may be altered with appropriate therapeutic intervention, repeated assessment is needed.

\section{References:}

[1] Kovacs G, Olschewski H. Borderline pulmonary pressures in scleraderma - "a pre-pulmonary arterial hypertension" condition? Arthritis Res Ther 2015; 17: 123, 015-0649-7.

[2] Kovacs G, Avian A, Tscherner M, et al. Characterization of patients with borderine pulmonary arterial pressure. Chest 2014; 146: 1486-93.

Disclosure of Interest: None declared

DOI: 10.1136/annrheumdis-2017-eular.3592

\section{SAT0370 EVALUATIONS OF EXERTION DYSPNEA IN PATIENTS WITH CONNECTIVE TISSUE DISEASE (CTD) BY CPET (CARDIOPULMONARY EXERCISE TESTING) FOR EARLY DETECTING ASSOCIATED PULMONARY HYPERTENSION (APAH)}

Y. Nobuhara, Y. Kamei, Y. Fujikawa, T. Nakazawa. Rheumatology, Osaka saiseikai nakatsu hospital, Osaka, Japan

Background: Patients with CTD often complain of exertion dyspnea, due to lung diseases, heart diseases, musculoskeletal disorders and/or APAH. Although imaging and physiological tests could reveal pathophysiology, some of them remain unknown. Especially APAH is rarely diagnosed in WHO functional class I or II, because pulmonary vasculopathy (PV) cannot be detected until two-thirds of pulmonary blood vessels deteriorated, ${ }^{1}$ with cardiac ultrasonography (UCG) or right heart catheterization (RHC) at rest. Pathophysiological considerations suggest that the haemodynamic/metabolic impairment in APAH may be observed during exercise before the disease becomes evident at rest. ${ }^{2-3}$ We evaluated exertion dyspnea of unknown to find out early APAH with CPET.

Objectives: We performed CPET in patients who complained of exertion dyspnea and tried to detect early PV.

Methods: From June 13th in 2015 to October 28th in 2016, we performed CPET and evaluate their clinical state in 28 patients, 17-80 years 2 males/26 females, 5 mixed connective tissue disease (MCTD), 15 SSc, 3 SLE, 2 Sjögren's syndrome, 3 dermatomyositis. They underwent UCG, pulmonary function testing (PFT), 6 -minutes walk test, and nailfold capillaroscopy by Cutolo's method. ${ }^{4}$

Results: Twenty cases presented decreased peak $\mathrm{VO} 2 . \mathrm{VE} / \mathrm{VCO}_{2}$ ratio, which represent increased ventilation-perfusion mismatch, elevated in 12 cases. 8 cases, with decreased peak $\mathrm{VO} 2$ but normal $\mathrm{VE} / \mathrm{VCO}_{2}$, were regarded that muscle weakness mainly induced exertion dyspnea, and advised exercise. Twelve cases with decreased peak VO2 and elevated $\mathrm{VE} / \mathrm{VCO}_{2}$ were estimated to have APAH or/and interstitial lung disease (ILD). Seven of them underwent RHC and 1 case diagnosed as definite APAH, and another 1 case as post capillary PH. A 34 years MCTD woman without ILD showed an active capillaroscopic pattern, her peak VO2 decreased $\left(13.9 \mathrm{ml} / \mathrm{kg} / \mathrm{min}\right.$.) and nadir $\mathrm{VE} / \mathrm{VCO}_{2}$ elevated (39). Although her mean PAP was normal, we suspected she had early PV and administered PDE5 inhibitor to her and her dyspnea had gone soon and CPET parameters improved.

CPET was also useful for early detections of therapeutic gains in APAH. A SSc woman, diagnosed as APAH by RHC, was performed CPET only 9 days after administration of PDE5 inhibitor. Her peak VO2 elavated (13.3 to $15.4 \mathrm{ml} / \mathrm{kg} / \mathrm{min}$.) and nadir $\mathrm{VE} / \mathrm{VCO}_{2}$ decreased (43.4 to 38.9) promptly.

Thirteen patients showed an early capillaroscopic pattern, 7 an active pattern and 3 a late pattern. Median values of minimum $\mathrm{VE} / \mathrm{VCO}_{2}$ ratio significantly differed $(p<0.05)$ in the three capillaroscopic groups, being progressively worse from early to late capillaroscopic pattern.

Conclusions: We performed CPET in every patient safely. Although more research is required, CPET may provide valuable information notably in APAH patients.

References:

[1] Hachulla E, et al. French PAH-SSc Network. PAH with SSc in patients with functional class? dyspnea; mild symptoms but severe outcome. Rheumatology 2010;49:940-4.

[2] Grunig E, et al. Non-invasive diagnosis of pulmonary hypertension: ESC/ERS Guidelines with Updated Commentary of the Cologne Consensus Conference 2011. Int J Cardiol 2011;154:53.

[3] Domitrescu C, et al. Developing PV in SSc, detected with non-invasive CPET. PLOS ONE 2010;5e14293.

[4] Cutolo M, et al. Nailfold videocapillaroscopy assessment of microvascular damage in SSc. J Rheumatol. 2000 Jan;27(1):155-60.

Disclosure of Interest: None declared

DOI: 10.1136/annrheumdis-2017-eular.4184

\section{SAT0371 BONE FRACTURE RISK ASSESSMENT IN PATIENTS WITH IDIOPATHIC INFLAMMATORY MYOPATHIES: RESEMBLANCES TO RHEUMATOID ARTHRITIS}

Z. Griger, A. Vincze, K. Szabó, J. Gaál, K. Dankó. Division of Clinical Immunology, Department of Internal Medicine, University of Debrecen, Debrecen, Hungary

Background: Idiopathic inflammatory myopathies are chronic, heterogeneous systemic autoimmune diseases with symmetrical proximal muscle weakness. During the disease course, osteoporosis and bone fractures are more common compared to the healthy population, which can be explained by the chronic inflammation, immobilization, spontaneous falls and steroid treatment, and affect crucially the patients' quality of life. Recently, a WHO fracture risk calculation tool, FRAX score is available, to measure the 10-year probability of osteoporotic fractures. It takes into account relevant clinical risk factors, such as rheumatoid arthritis, however myositis does not exist among the risk factors.

Objectives: Estimation the effect of myositis and myositis related bone mineral densities on bone fracture risk calculated by FRAX tool.

Methods: FRAX score was determined in 71 patients with idiopathic inflammatory myopathies and results were compared with the data from 50 age, sex and BMI matched patients with rheumatoid arthritis. Moreover, osteoporosis related biomarkers, disease related fractures and bone mineral densities were determined using DXA examinations. Statistical analysis was performed with IBM SPSS 20.0 software.

Results: There were no significant differences between the demographical data, biomarkers (Ca, Vitamin D, parathormone level) of the two groups. Disease duration and cumulative steroid dose were higher in the myositis group. Results of the FRAX score without BMD were significantly lower in the patients with myositis, in both fracture risk: major osteoporotic $(8,61 \pm 6,36 \%$, vs. $15,59 \pm 12,66 \%$; p: $0,002)$ and femur neck $(2,66 \pm 3,24 \%$, vs. $6,34 \pm 9,018 ; p$ : 0.003$)$. T score results of the DXA examination were not significantly different between the two populations (Lumbar1-4: $-0,9 \pm 1,43$ vs. $-0,829 \pm 1,38$; p:0,829; Femoral neck: $-1,4 \pm 1,08$ vs. $-1,02 \pm 1,08$; p: 0,93$)$, but the presence of osteopenia ( $60 \%$ vs. $39,5 \%)$ and osteoporosis $(13,5 \%$ vs. $7 \%$ ) were more frequent in the myositis group (p: 0,045$)$. Disease related fracture was associated with disease duration in the myositis group and with antibody (RF, ACPA) presence in the RA group. FRAX score with BMD results showed no significant differences between the two populations

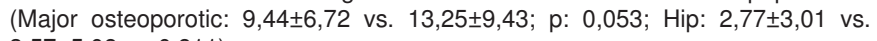
3,57士5,08; p: 0,811).

Conclusions: As far as we know, this is the first study which examine the fracture risk using FRAX score in patients with idiopathic inflammatory myopathies. According to our data, we can conclude that existence of myositis might indicate similar, independent risk factor in fracture probability, like rheumatoid arthritis. Evaluation of fracture risk should be done with DXA result in patients with IIM, otherwise risk could be underestimated. An exact value of the "myositis related risk" could be determined by a 10-year prospective study.

Disclosure of Interest: None declared

DOI: 10.1136/annrheumdis-2017-eular.3784

\section{SAT0372 CLINICAL AND SEROLOGICAL ASSOCIATIONS OF AUTOANTIBODIES TO BICD2 AS A NOVEL MARKER FOR SYSTEMIC SCLEROSIS}

M. Mahler ${ }^{1}$, C. Bentow ${ }^{1}$, J. Milo ${ }^{1}$, M. Hudson ${ }^{2}$, H.-D. Zucht ${ }^{3}$, P. Budde ${ }^{3}$, D. Wirtz ${ }^{3}$, J. Schulte-Pelkum ${ }^{3}, \mathrm{M}$. Choi ${ }^{4}, \mathrm{M}$. Fritzler ${ }^{4} .{ }^{1}$ Research and Development, Inova Diagnostics, San Diego, CA., United States; ${ }^{2}$ Department of Medicine, McGill University, Quebec, Canada: ${ }^{3}$ Protagen, Dortmund, Germany; ${ }^{4}$ Cumming School of Medicine, University of Calgary, Calgary, Canada

Background: Anti-nuclear antibodies (ANA), which are present in approximately $90 \%$ of systemic sclerosis (SSc) patient's sera, play an important role in establishing the diagnosis and predicting prognosis of SSc. Recently, a novel autoantibody has been described in SSc patients targeting Cytoskeleton-Like Bicaudal D Protein Homolog 2 (BICD2).

Objectives: The aim of this study was to assess the prevalence and titers of anti-BICD2 antibodies in SSc and controls and to study the clinical associations of this new antibody.

Methods: A total of 502 samples from SSc patients enrolled in the Canadian Scleroderma Research Group (CSRG) cohort were included in this study. Clinical associations were assessed either as anti-BICD2 antibody positivity 